\title{
A Membrane-Type Acoustic Metamaterial With Self-Similar Structure
}

\author{
Yingxu Fan, Zhimin Xie*, Yuhong Qie \\ National Key Laboratory of Science and Technology on Advanced Composites in Special Environment, Center for Composite \\ Materials, Harbin Institute of Technology, Harbin 150001, P.R. China
}

Keywords: Self-similar, acoustic metamaterial, locally resonant, band-gap.

\begin{abstract}
The locally resonant membrane-type metamaterial has a unique feature of narrow frequency band-gaps where the waves can't propagate, therefore it would be not suitable for broadband noise reduction. To broaden the frequency bandgap, we proposed a self-similar structure with ring mass for the membrane-type metamaterial in the present work. The dispersion curves of the self-similar structures under equalbiaxial tension were obtained by using finite element method. It is found that the number of band-gap increases with an increase of the ring mass. In this sense, the frequency bandgap is broadened. The analysis of harmonic response revealed that the anti-resonant frequency was just located in the first band-gap in the dispersion curve diagram. For this antiresonant mode of the local membrane-mass/ring mass resonator, the ring mass vibrated out-of-phase with the membrane, therefore it is anticipated that a transmission loss (TL) peak would appear in the TL profile.
\end{abstract}

\section{Introduction}

It has been known that the sound attenuation can be enhanced by increasing the structure thickness. According to the conventional mass-density law in the absence of absorption, a light-weight structure find ineffective in attenuating sound at low frequency. In last two decades there has been considerable interest in the study of low-frequency acoustic filter with the metamaterials since the locally resonant metamaterials have been shown to break the conventional mass-density law ${ }^{[1-9]}$. Although the locally resonant metamaterials have positive static elastic constants, their effective elastic constants and/or dynamic mass density are negative at certain frequency ranges.

Membrane-type acoustic metamaterial, constructed of one or more cells, is one of the most important locally resonant metamaterials. Generally, one unit cell of the membrane-type acoustic metamaterial consists in a rigid frame and a thin, tensioned membrane carrying a concentrated mass. Many researchers have demonstrated that the membrane-type acoustic metamaterials can enhance sound insulation at low frequencies ${ }^{[1-9]}$. Naify et al. ${ }^{[2-5]}$ carried out a series of experiments on the metamaterials to investigate the effects of attaching mass weight, membrane tension, frame stiffness and multiple elements arranged in arrays on the transmission loss (TL) and dynamic response. The results of the measurement using an impedance tube indicated that the acoustic metamaterials had a significant increase in TL in comparison with the mass law predictions at a range of $100-1000 \mathrm{~Hz}$. Based on measurement of the displacement response of the structure at the peak TL frequency and the pressureacceleration analysis, they obtained the negative effective mass at the peak TL frequency. According to the analysis of Zhang et al. ${ }^{[10]}$, the first TL valley and peak depended strongly on the attaching mass, while the second TL valley was mainly influenced by the membrane properties. To study the sound transmission behavior and accurately capture the rigid mass effects on the membrane deformation, Chen et al. ${ }^{[11]}$ have recently developed an analytical vibroacoustic membrane model using the point matching approach by applying a set of distributed point forces along the interfacial boundary between masses and the membrane. In view that total reflection for the elastic wave or sound occurs at the anti-resonant frequencies and near-complete transmission occurs at the resonant frequencies for the membrane-type acoustic metamaterials, Ma et al. ${ }^{[7]}$ proposed a low-frequency narrow-band acoustic filter with a large orifice, by which large TL was achieved across the orifice. Furthermore, by exploiting properties of membrane-type acoustic metamaterials, Ma et al. ${ }^{[8]}$ designed and fabricated an acoustic metasurface with a decorated membrane resonator, a reflecting surface, and a thin sealed gas layer in between, and then achieved robust impedance matching and perfect lowfrequency absorption.

The locally resonant membrane-type metamaterial has a unique feature of narrow frequency band-gaps where the waves can't propagate, therefore it would be not suitable for broadband noise reduction. To broaden the frequency bandgap, we proposed a self-similar structure for the membranetype metamaterial in the present work. The band-gap properties were investigated by using finite element method.

\section{Self-s imilar structure}

The membrane-type metamaterial is a periodic structure. One unit cell of the membrane-type metamaterial consists of a square membrane stretched in equal-biaxial tension, a support frame, and a group of ring mass attached to the membrane, as schematically shown in Figure 1. In the self-similar structure, the ratios of outer side to inner side of the ring are kept constant, i.e.,

\footnotetext{
* Corresponding Author
} 


$$
\frac{d_{\text {lout }}}{d_{\text {lin }}}=\frac{d_{2 \text { out }}}{d_{2 \text { in }}}=\ldots=\frac{d_{\text {iout }}}{d_{\text {iin }}}=\frac{D}{d}
$$

where the subscripts of "out" and "in" denote the outer side and inner side, respectively. D and $d$ are the length of outer side and inner side of the frame, respectively. It is assumed that the central mass and the rings have the material density $\rho$, and all the rings have the same weight as the central mass, which can be reached by adjusting the height of the rings.

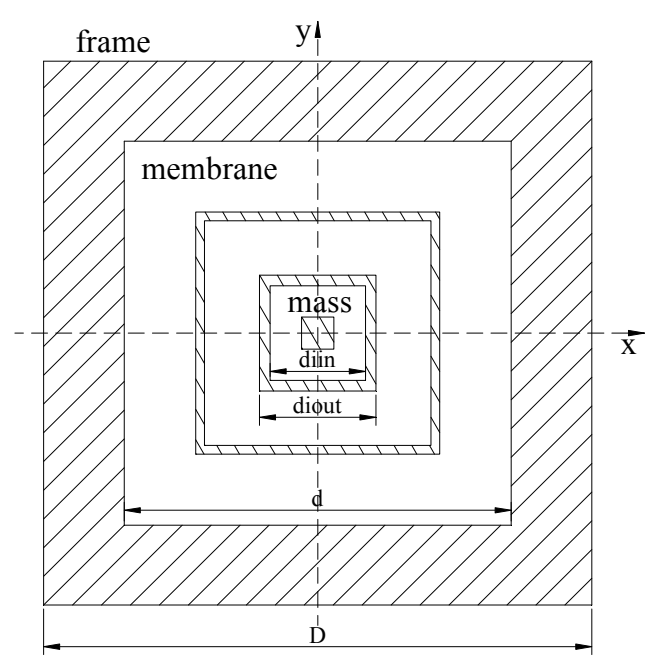

Figure1: Schematic diagram of one unit of the membranetype metamaterial

\section{Computational method for band-gap characteristics}

Base upon the Kirchhoff plate theory, the differential equation of motion of a unit cell of the frame-membrane-mass system in absence of ring mass under equal-biaxial tension $\mathrm{f}$ is given by,

$$
\begin{aligned}
& B_{F} \hbar_{1} \nabla^{4} w+B_{m} \hbar_{3} \nabla^{4} w-f \frac{\partial^{2} w}{\partial x^{2}}-f \frac{\partial^{2} w}{\partial y^{2}} \\
& \quad+\left(\rho_{F} \hbar_{1} h_{F}+\rho_{\text {mass }} \hbar_{2} h_{\text {mass }}+\rho_{m} \hbar_{3} h_{m}\right) \ddot{w}=0
\end{aligned}
$$

where $\mathrm{w}$ is the transversal displacement, $\rho$ is the mass density, $\mathrm{h}$ is the thickness, a superimposed dot means time-derivative, subscripts of "F", "m", and "mass" denote the frame, membrane, and mass, respectively. $\mathrm{B}$ is the flexural rigidity determined by

$$
B=\frac{E h^{3}}{12\left(1-v^{2}\right)}
$$

with the elastic modulus E and Poisson's coefficient $v$ of the material. $\hbar$ is related to the Heaviside function $\mathrm{H}(\mathrm{)})$ as listed in Appendix.

According to the finite element solution process by Gei et al. ${ }^{[12]}$, a weak formulation of dynamics was obtained by,

$$
\begin{aligned}
& \int_{\Omega} A^{T} C A d \Omega-\left(\rho_{F} \hbar_{1}+\rho_{m} \hbar_{3}+\rho_{\text {mass }} \hbar_{2}\right) \\
& \cdot \omega^{2} \int_{\Omega} w^{2} d \Omega+\int_{\Omega} f\left[\left(\frac{\partial w}{\partial x}\right)^{2}+\left(\frac{\partial w}{\partial y}\right)^{2}\right] d \Omega=0
\end{aligned}
$$

in which,

$$
\begin{aligned}
& A=\left(\frac{\partial^{2} w}{\partial x^{2}}, \frac{\partial^{2} w}{\partial y^{2}}, 2 \frac{\partial^{2} w}{\partial x \partial y}\right)^{T}, \\
& C=B\left[\begin{array}{ccc}
1 & v & 0 \\
v & 1 & 0 \\
0 & 0 & (1-v) / 2
\end{array}\right] .
\end{aligned}
$$

A finite element(FE) discretization was made at the domain $\Omega$ by using the quadratic element. The transversal displacement w can be expressed in term of $u^{e}$ (nodal displacements and rotations) as

$$
w=N(x, y) \cdot u^{e}
$$

with a shape function $\mathrm{N}(\mathrm{x}, \mathrm{y})$ which can be found in the FE textbook.

Substitution of Eq.(6) into Eq.(3) yields,

$$
u^{e} \cdot\left(K_{e}+K_{f e}-\omega^{2} M_{e}\right) u^{e}=0
$$

in which,

$$
\begin{aligned}
& M_{e}=\int_{\Omega^{e}}\left(\rho_{\text {mass }} h_{\text {mass }} \hbar_{2}+\rho_{F} h_{F} \hbar_{1}+\rho_{m} h_{m} \hbar_{3}\right) \text {, } \\
& \cdot N(x, y)^{T} N(x, y) d \Omega^{e} \\
& K_{e}=\int_{\Omega^{e}} P^{T} C P d \Omega^{e}, \\
& K_{f e}=\int_{\Omega^{e}} P_{f}^{T} C_{f} P_{f} d \Omega^{e}, \\
& P=\left(\frac{\partial^{2} N(x, y)^{T}}{\partial x^{2}}, \frac{\partial^{2} N(x, y)^{T}}{\partial y^{2}}, 2 \frac{\partial^{2} N(x, y)^{T}}{\partial x \partial y}\right)^{T}, \\
& P_{f}=\left(N(x, y)^{T}, \frac{\partial N(x, y)^{T}}{\partial x}, \frac{\partial N(x, y)^{T}}{\partial y}\right)^{T} \text {, } \\
& C_{f}=\left(\begin{array}{ccc}
0 & 0 & 0 \\
0 & f & 0 \\
0 & 0 & f
\end{array}\right) \text {. }
\end{aligned}
$$

Furthermore, the eigen value problem governing timeharmonic vibrations for the whole cell domain can be obtained by assembling the matrix of the elements, i.e.,

$$
u \cdot\left(K+K_{f}-\omega^{2} M\right) u=0
$$

where $\mathrm{K}, \mathrm{K}_{\mathrm{f}}$, and $\mathrm{M}$ are the whole matrices by assembling the matrices $\mathrm{K}_{\mathrm{e}}, \mathrm{K}_{\mathrm{fe}}$, and $\mathrm{M}_{\mathrm{e}}$, respectively. Appling the FloquetBloch condition for the unit cell of the periodic system yields, $w(x+m D, y+n D)=w(x, y) e^{i\left(k_{1} m D+k_{2} n D\right)}$

$u(D, y)=u(0, y) e^{i k_{1} D}, \quad 0 \leq y \leq D$ 


$$
u(x, D)=u(x, 0) e^{i k_{2} D}, \quad 0 \leq x \leq D
$$

where $\mathrm{m}$ and $\mathrm{n}$ are integers indexing a node of the cell and $\mathrm{k}=$ $\left(\mathrm{k}_{1}, \mathrm{k}_{2}\right)$ is the Bloch vector. Application of the periodic boundary condition leads to a final form of the eigen value problem as follows,

$$
\left(\breve{K}+\tilde{K}_{f}-\omega^{2} \tilde{M}\right) u=0
$$

where $\widetilde{u}$ is the linearly independent components of displacement along the boundary and $u=T(k) \widetilde{u} . \widetilde{K}, \widetilde{K}_{f}$ and $\tilde{M}$ are determined by $K, K_{f}, M$ and the transformation matrix $T(k)$. A Matlab code was developed to calculate the eigen frequency. When the Bloch vector is selected along the edge $\Gamma-\mathrm{M}-\mathrm{X}-\Gamma$ of the first irreducible Brillouin zone, as depicted in Figure 2, a series of dispersion curves of the periodic system can be obtained.

To examine the dynamic characteristics of the self-similar system, the analysis of harmonic response was performed using a commercial software Abaqus.

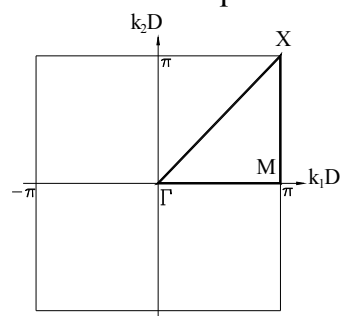

Figure 2: The first irreducible Brillouin zone

\section{Results and discussion}

The self-similar metamaterial was designed based upon one unit cell of frame-membrane-mass system, or namely basic system, with $\mathrm{D}=85 \mathrm{~cm}, \mathrm{~d}=60 \mathrm{~cm}, \quad a=5 \mathrm{~cm}, \rho_{\mathrm{F}}=0.59 \mathrm{~kg} / \mathrm{m}^{2}$, $\rho_{\mathrm{m}}=0.49 \mathrm{~kg} / \mathrm{m}^{2}, \rho_{\text {mass }}=11.99 \mathrm{~kg} / \mathrm{m}^{2}, \mathrm{E}_{\mathrm{F}}=10 \mathrm{GPa}, \mathrm{E}_{\mathrm{m}}=0.2 \mathrm{MPa}$, $v_{\mathrm{F}}=0.3, v_{\mathrm{m}}=0.49$, and $\mathrm{h}_{\mathrm{m}}=0.5 \mathrm{~mm}$. The dispersion curves were computed using the Matlab code mentioned above for the basic system, the basic system with two and three ring masses along the edge $\Gamma-\mathrm{M}-\mathrm{X}-\Gamma$ of the first irreducible Brillouin zone, as shown in Figure 3, where the prestress is 500Pa. It is found that there is only one complete band-gap in the range of 0 $80 \mathrm{~Hz}$ for the basic system. The number of band-gap at the same frequency range increases with an increase of the ring mass, for instance, three band-gaps appear in the case of three ring masses, for that an introduction of the ring mass leads to an increase of modes of vibration. In this sense, the frequency band-gap is broadened.

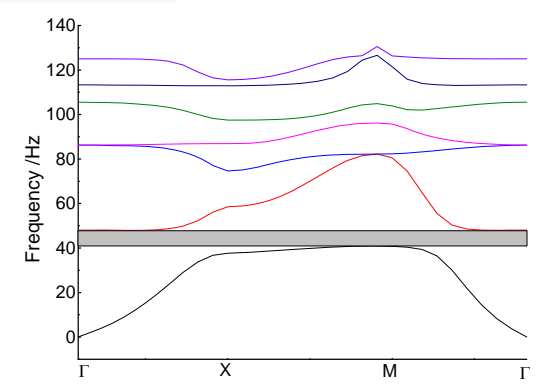

(a) basic system

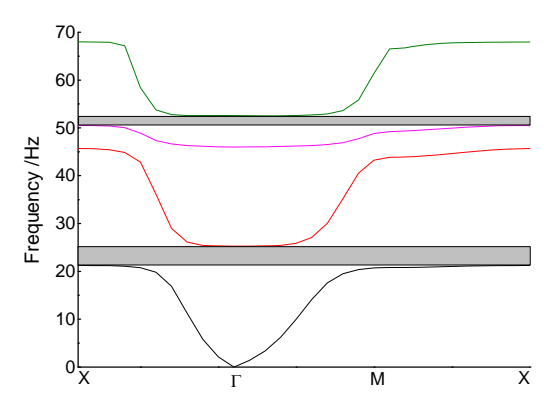

(b) basic system with two ring masses

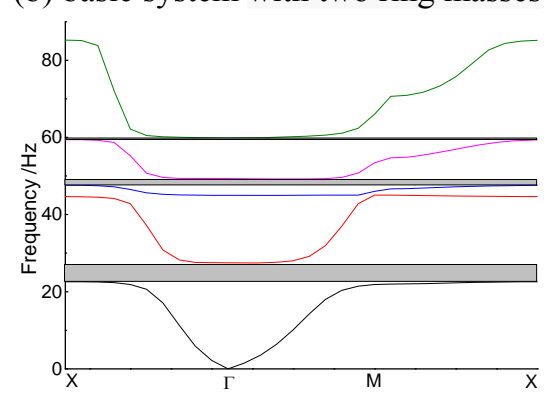

(c) basic system with three ring masses

Figure 3: Dispersion curves.

In the membrane-type acoustic metamaterials, the prestress plays an important role in the dynamic properties. Figure4 shows variation of the central frequency of the first band-gap with the prestress. Clearly, the central frequency increases with increasing the prestress, because the prestress improves the transversal stiffness of the membrane. Additionally, the basic system has a highest central frequency of the first bandgap than the basic system with one and two ring masses.

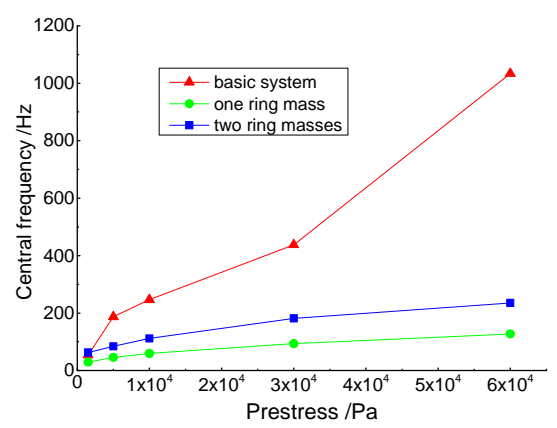

Figure 4: Variation of the central frequency of the first bandgap with the prestress.

The displacement response of the basic system with two ring masses was computed by using the software Abaqus to examine the dynamic characteristics of the self-similar system. The harmonic vibration of the system was under the harmonic loading excited on the central mass. Five points were selected along $\mathrm{x}$ axial. Point 1 lies in the frame, points 2 and 3 in the ring mass, point 4 in the mass, and point 5 in the membrane.

The displacements of five points were recorded, as shown in Figure 5. As can be seen, there are two resonant band, one at around $7 \mathrm{~Hz}$ and the other at around $24 \mathrm{~Hz}$. In fact, the second resonant frequency is just located in the first band-gap as shown in Figure 3(b). The second mode was associated with the internal resonance of the elastic motion of the membrane. For the anti-resonant mode of the local membrane-mass/ring 
mass resonator, the ring mass vibrated out-of-phase with the membrane. According to the analysis of Zhang et al. ${ }^{[10]}$, it is anticipated that a TL peak would appear in the TL profile.
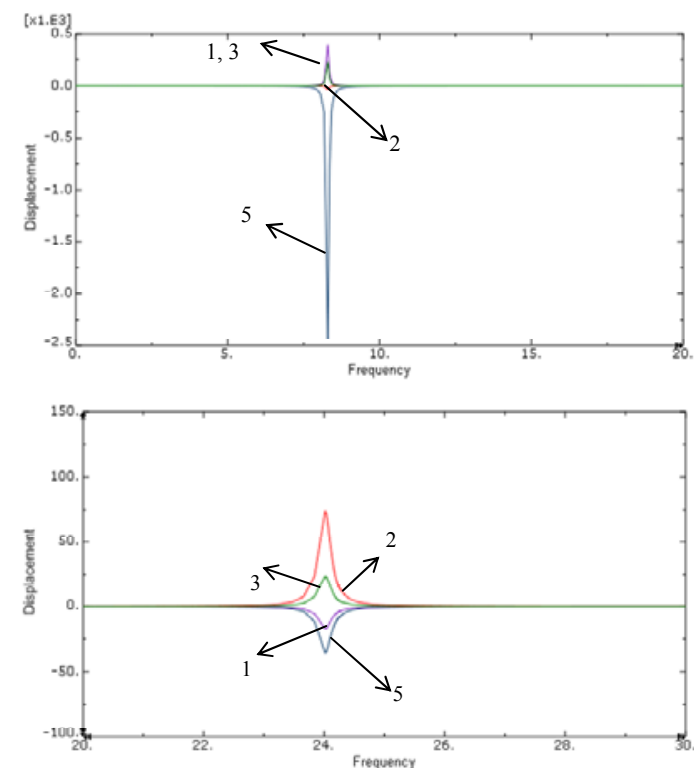

Figure 5: The displacement response of self-similar system with two ring masses

\section{Conclusions}

A self-similar structure with ring mass for the membrane-type metamaterial was proposed. The dispersion curves of selfsimilar structures under equal-biaxial tension were obtained by using finite element method. It is found that the number of band-gap increases with an increase of the ring mass, for that an introduction of the ring mass leads to an increase of modes of vibration. In this sense, the frequency band-gap is broadened. The analysis of harmonic response revealed that the second resonant mode was associated with the internal resonance of the elastic motion of the membrane, whose frequency was just located in the first band-gap in the dispersion curve diagram. For this anti-resonant mode of the local membrane-mass/ring mass resonator, the ring mass vibrated out-of-phase with the membrane, therefore it is anticipated that a TL peak would appear in the TL profile.

\section{Acknowledgements}

The authors gratefully acknowledge financial support by the National Science Foundation of China under Projects No.11272104.

\section{Appendix}

$$
\begin{gathered}
\hbar_{1}=\{H[(D-d) / 2-x]+H[x-(D+d) / 2]\} \\
+\{H[(D-d) / 2-y]+H[y-(D+d) / 2]\} \\
+\{H[(D-d) / 2-x]+H[x-(D+d) / 2]\} \\
\quad \cdot\{H[(D-d) / 2-y]+H[y-(D+d) / 2]\}
\end{gathered}
$$

$$
\begin{gathered}
\hbar_{2}=\{H[x-(D-a) / 2]-H[x-(D+a) / 2]\}, \\
\cdot \cdot H[y-(D-a) / 2]+H[y-(D+a) / 2]\}, \\
\hbar_{3}=\{H[x-(D-d) / 2]-H[x-(D+d) / 2]\}, \\
\cdot \cdot H[y-(D-d) / 2]+H[y-(D+d) / 2]\}
\end{gathered},
$$

where $a$ is the length of the mass.

\section{References}

[1] Z. Liu, X. Zhang, Y. Mao, et al. "Locally Resonant Sonic Materials", Science 289, pp. 1734-1736, (2000).

[2] C. J. Naify, C. M. Chang, G. McKnight, et al. "Transmission loss and dynamic response of membranetype locally resonant acoustic metamaterials", Journal of Applied Physics, 108, pp. 114905, (2010).

[3] C. J. Naify, C. M. Chang, G. McKnight, et al. "Transmission loss of membrane-type acoustic metamaterials with coaxial ring masses", Journal of Applied Physics, 110, pp. 124903, (2011).

[4] C. J. Naify, C. M. Chang, G. McKnight, et al. "Scaling of membrane-type locally resonant acoustic metamaterial arrays", The Journal of the Acoustical Society of America 132, pp. 2784-2792, (2012).

[5] C. J. Naify, C. M. Chang, G. McKnight, et al. " Membrane-type metamaterials: Transmission loss of multi-celled arrays", Journal of Applied Physics, 109, pp. 104902, (2011).

[6] J. S. Chen, D. W. Kao. "Sound Attenuation of Membranes Loaded with Square Frame-Shaped Masses", Mathematical Problems in Engineering 2016, pp. 1740236, (2016).

[7] G. Ma, M. Yang, Z. Yang, et al. "Low-frequency narrow-band acoustic filter with large orifice", Applied Physics Letters 103, pp. 011903, (2013).

[8] G. Ma, M. Yang, S. Xiao, et al. " Acoustic metasurface with hybrid resonances ", Nature Materials 13, pp. 873878, (2014).

[9] J. Mei, G. Ma, M. Yang, et al. "Dark acoustic metamaterials as super absorbers for low-frequency sound", Nature Communications 3, pp. 756, (2012).

[10] Y. Zhang, J. Wen, Y. Xiao, et al. "Theoretical investigation of the sound attenuation of membrane-type acoustic metamaterials", Physics Letters A 376, pp. 1489-1494, (2012).

[11] Y. Chen, G. Huang, X. Zhou, et al. "Analytical coupled vibroacoustic modeling of membrane-type acoustic metamaterials: Membrane model", The Journal of the Acoustical Society of America 136, pp. 969-979, (2014).

[12] M. Gei, D. Bigoni, A. B. Movchan, et al. "Band-Gap Properties of Prestressed Structures", in Acoustic Metamaterials, Ed. by R. V. Craster and S. Guenneau. Springer, pp. 61-82, (2013) 